VOL. 48 (1993) [1-6]

\title{
COUNTABLY QUASI-SUPRABARRELLED SPACES
}

\author{
J.C. Ferrando and L.M. Sánchez Ruiz
}

In this paper we obtain some permanence properties of a class- of locally convex spaces located between quasi-suprabarrelled spaces and quasi-totally barrelled spaces, for which a closed graph theorem is given.

\section{INTRODUCTION}

Throughout this paper the word "space" will stand for "Hausdorff locally convex topological vector space defined over the field $\mathbb{K}$ of real or complex numbers". Let us recall a space $E$ is quasi-suprabarrelled [1] if, given an increasing sequence of subspaces of $E$ covering $E$, there is one which is barrelled; $E$ satisfies condition (G) [4] if, given a sequence of subspaces of $E$ covering $E$, there is one which is barrelled; $E$ is quasitotally barrelled [2] if, given a sequence of subspaces of $E$ covering $E$, there is one which is barrelled and its closure has countable codimension in $E ; E$ is totally barrelled [12] if, given a sequence of subspaces of $E$ covering $E$, there is one which is barrelled and its closure has finite codimension in $E ; E$ is unordered Baire-like [6] if, given a sequence of closed absolutely convex subsets of $E$ covering $E$, there is one which is a neighbourhood of the origin; and $E$ is suprabarrelled [9] ((bd) in [5]) if, given an increasing sequence of subspaces of $E$ covering $E$, there is one which is barrelled and dense in $E$. The relationship among these classes of spaces is the following:

unordered Baire-like $\Rightarrow$ totally barrelled $\Rightarrow$ suprabarrelled $\Rightarrow$ barrelled.

and

totally barrelled $\Rightarrow$ quasi-totally barrelled $\Rightarrow(G) \Rightarrow$ quasi-suprabarrelled $\Rightarrow$ barrelled.

In this paper we shall introduce a class of spaces located between quasi-totally barrelled spaces and quasi-suprabarrelled spaces, which enjoys good permanence properties, and satisfies a closed graph theorem.

Our notation is standard, so if $A$ is a subset of a linear space, $\langle A\rangle$ will denote its linear span and if $\left\{E_{i}: i \in I\right\}$ is a family of spaces, $E=\Pi\left\{E_{i}: i \in I\right\}$ and $J$ is a subset of $I, E(J)$ will denote the subspace of $E$ consisting of those elements of support $J$.

Received 30 June 1992

Copyright Clearance Centre, Inc. Serial-fee code: 0004-9729/93 \$A2.00+0.00. 


\section{COUNTABLY QUASI-SUPRABARRELLED SPACES}

Definition: We shall say a space $E$ is countably quasi-suprabarrelled if, given an increasing sequence of subspaces $\left\{E_{n}: n \in \mathbb{N}\right\}$ covering $E$, there is one of them, say $E_{p}$, which is barrelled and its closure, $\overline{E_{p}}$, has countable codimention in $E$.

Clearly, quasi-totally barrelled $\Rightarrow$ countably quasi-suprabarrelled $\Rightarrow$ quasisuprabarrelled, and suprabarrelled $\Rightarrow$ countably quasi-suprabarrelled.

It is easy to check that if $c$ is the cardinal of the continuum, $\varphi_{c}$ verifies condition (G) and, consequently, is quasi-suprabarrelled but not countably quasi-suprabarrelled, [2, Example 2]. On the other hand, $\varphi$ is a non-suprabarrelled countably quasisuprabarrelled space since every linear subspace of it has countable codimension. Moreover, if $I$ is any index set, it follows from Theorem 1 below that $\varphi^{I}$ is also a nonsuprabarrelled countably quasi-suprabarrelled space, and, in general, each countably quasi-suprabarrelled space containing a complemented copy of $\varphi$ is not suprabarrelled.

EXAMPLES. Countably quasi-suprabarrelled spaces which are not quasi-totally barrelled.

1. Let $E$ be a locally convex space and $m_{0}(E)$ the space of the $2^{\mathbf{N}}$-simple functions defined over $\mathbb{N}$ with values in $E$ endowed with the uniform convergence topology. From [3] it follows that if $E$ is nuclear and unordered Baire-like, then $m_{0}(E)$ is suprabarrelled and, consequently, countably quasi-suprabarrelled. If $\left\{A_{n}: n \in \mathbb{N}\right\}$ denotes the sequence of all the subsets of two different positive integers of $\mathbb{N}$ and $L_{n}$ is the linear subspace of $m_{0}(E)$ of all the $f \in m_{0}(E)$ which are constant on $A_{n}$, it is clear that each $L_{n}$ is closed in $m_{0}(E)$, that $\left\{L_{n}: n \in \mathbb{N}\right\}$ covers $m_{0}(E)$ and that if $\operatorname{dim} E$ is uncountable, then each $L_{n}$ has uncountable codimension in $m_{0}(E)$. Hence, if $E$ is a nuclear unordered Baire-like space of uncountable dimension, $m_{0}(E)$ is a countably quasi-suprabarrelled space which is not quasi-totally barrelled.

2. Let $E$ be a Banach space containing a sequence of closed linear subspaces $\left\{X_{n}: n \in \mathbb{N}\right\}$ of infinite dimension such that for each $n \in \mathbb{N}$, the closed linear hull of $\left\{X_{m}: m>n\right\}$ is a topological complement of $X_{1}+\ldots+X_{n}$ and let $E_{n}$ be the closed linear hull of $\left\{X_{m}: m \in \mathbb{N} \backslash\{n\}\right\}$. If $\mathcal{U}$ is an ultrafilter in $\mathbb{N}$ which contains the filter of all the subsets of $\mathbb{N}$ whose complement has zero density, $L(U)$ the closure in $E$ of the linear hull of $\bigcup\left\{X_{n}: n \in \mathbb{N} \backslash U\right\}$ for each $U \in \mathcal{U}$, and $L:=\bigcup\{L(U): U \in \mathcal{U}\}$, then $L$ is a suprabarrelled and dense subspace in $E,\left[11\right.$, Proposition 12]. If each $X_{n}$ has infinite dimension, then $E_{n} \cap L$ is a subspace of uncountable codimension in $L$. Finally, as each $E_{n}$ is closed and $\left\{E_{n} \cap L: n \in \mathbb{N}\right\}$ covers $L$, we obtain that $L$ is a countably quasi-suprabarrelled space which is not quasi-totally barrelled.

Clearly, the topological product of $\varphi$ and any non-quasi-totally barrelled countably quasi-suprabarrelled space is an example of a countably quasi-suprabarrelled space 
which is neither suprabarrelled nor quasi-totally barrelled. On the other hand, a metrisable space $E$ is countably quasi-suprabarrelled if and only if $E$ is suprabarrelled. But, as we have mentioned above, there exist non-suprabarrelled countably quasi-suprabarrelled spaces. Next we shall show the following.

Proposition 1. Let $E$ be a countably quasi-suprabarrelled space. If $E$ is not suprabarrelled then $E$ is not Baire-like either.

Proof: If $E$ is not suprabarrelled, there exists an increasing sequence of linear subspaces $\left\{E_{n}: n \in \mathbb{N}\right\}$ of $E$ covering $E$, such that no $E_{n}$ is barrelled and dense at the same time. As $E$ is countably quasi-suprabarrelled, we may suppose that each $E_{n}$ is barrelled and its closure is of countable codimension. Hence $E$ cannot be Baire-like since it may be covered by an increasing sequence of closed linear subspaces of infinite countable codimension.

\section{Properties of COUNTABLY QUASI-SUPRABARRELlED SPACES}

Next we shall obtain some permanence properties of countably quasi-suprabarrelled spaces.

Proposition 2. Let $E$ be a countably quasi-suprabarrelled space. If $F$ is a closed linear subspace of $E$ then $E / F$ is countably quasi-suprabarrelled.

Proof: Let $\left\{G_{n}: n \in \mathbb{N}\right\}$ be an increasing sequence of subspaces of $E / F$ covering $E / F$. Let $k$ be the canonical mapping of $E$ onto $E / F$. Then $\left\{k^{-1}\left(G_{n}\right): n \in \mathbb{N}\right\}$ is an increasing sequence of subspaces of $E$ covering $E$, so there must be some some $p \in \mathbb{N}$ such that $k^{-1}\left(G_{p}\right)$ is barrelled and $\operatorname{cod}_{E} \overline{k^{-1}\left(G_{p}\right)} \leqslant \aleph_{0}$. Now, $G_{p}=k\left(k^{-1}\left(G_{p}\right)\right)$ is barrelled and if $L$ is an algebraic complement of $\overline{k^{-1}\left(G_{p}\right)}$ in $E$, then $G_{p}+k(L)=$ $\overline{k\left(k^{-1}\left(G_{p}\right)\right)}+k(L) \supset k\left(\overline{k^{-1}\left(G_{p}\right)}\right)+k(L)=k\left(\overline{k^{-1}\left(G_{p}\right)}+L\right)=k(E)=E / F$. Hence $\overline{G_{p}}$ has countable codimension in $E / F$.

Proposition 3. Let $F$ be a dense linear subspace of $E$. If $F$ is countably quasi-suprabarrelled then $E$ is countably quasi-suprabarrelled.

Proof: Let $\left\{E_{n}: n \in \mathbb{N}\right\}$ be an increasing sequence of subspaces of $E$ covering $E$. Since $F$ is countably quasi-suprabarrelled there is some $p \in \mathbb{N}$ such that each $F \cap E_{p}$ is barrelled and $\operatorname{cod} F \overline{F \cap E_{p}} F \leqslant \aleph_{0}$. Let $L$ be a topological complement of $\overline{F \cap E_{p}} F$ in $F . \overline{F \cap E_{p}} \oplus L$ coincides with $E$ since it is closed and $F \subset \overline{F \cap E} \oplus L$, so $\overline{E_{p}}+L=E$ and $\operatorname{cod}_{E} \overline{E_{p}} \leqslant \aleph_{0}$. Besides, $F \cap E_{p} \oplus_{t} L$ is a barrelled dense subspace of $E_{p}+L$. Hence $E_{p}$ is barrelled.

PROPOSITION 4. Let $F$ be a countable codimensional subspace of $E$. If $E$ is countably quasi-suprabarrelled then $F$ is countably quasi-suprabarrelled. 
Proof: Let $\left\{F_{n}: n \in \mathbb{N}\right\}$ be an increasing sequence of subspaces of $\boldsymbol{F}$ covering $F$. Let $G$ be an algebraic complement of $F$ in $E$. As $\left\{F_{n}+G: n \in \mathbb{N}\right\}$ is an increasing sequence of subspaces of $E$ covering $E$, we may assume that every $F_{n}$ is barrelled. On the other hand, as $\left\{\overline{F_{n}}+G: n \in \mathbb{N}\right\}$ is also an increasing sequence of subspaces of $E$ covering $E$ we may assume that every $\overline{F_{n}}+G$ is barrelled. So, if $L_{n}$ is a topological complement of $\overline{F_{n}}$ in $\overline{F_{n}}+G, L_{n} \cong \varphi,[7]$. Therefore, $\overline{F_{n}}+G=\overline{F_{n}} \oplus_{t} L_{n}$ is a closed subspace of $E$ for every $n \in \mathbb{N}$ and there must be some $p \in \mathbb{N}$ so that $\overline{F_{p}}+G$, and consequently $\overline{F_{p}}$, has countable codimension in $E$. Hence $\overline{F_{p}}$ F has countable codimension in $F$.

PropostTION 5. The topological product of finitely many countably quasisuprabarrelled spaces is countably quasi-suprabarrelled.

Proof: Assume $E_{1}$ and $E_{2}$ are countably quasi-suprabarrelled and $E=E_{1} \times E_{2}$. Let $\left\{F_{n}: n \in \mathbb{N}\right\}$ be an increasing sequence of subspaces of $E$ covering $E$. Then there exists a subsequence $\left\{F_{n_{p}}: p \in \mathbb{N}\right\}$ such that $\operatorname{cod}_{E_{i}}{\overline{F_{n_{p}} \cap E_{i}}}_{E_{\mathbf{i}}} \leqslant \aleph_{0}, i=1,2$. For each $p \in \mathbb{N}$ let $A_{p, i}$ be a cobasis of $\overline{F_{n_{p}} \cap E_{i}} E_{i}$ in $E_{i}, i=1,2$. Set $A:=\bigcup\left\{A_{p, 1} \cup A_{p, 2}: p \in\right.$ $\mathbb{N}\}$ and, for each $p \in \mathbb{N}$, let $L_{p}:=\left\langle F_{n_{p}} \cup A\right\rangle$. If some $L_{p}$ were barrelled, $F_{n_{p}}$ would be barrelled and the proof would be finished since $E=E_{1} \times E_{2}=\left(\overline{F_{n_{p}} \cap E_{1}} E_{1}+\left\langle A_{p, 1}\right\rangle\right) \times$ $\left({\overline{F_{n_{p}} \cap E_{2}}}^{E_{2}}+\left\langle A_{p, 2}\right\rangle\right) \subset{\overline{F_{n_{p}}}}^{E}+\left\langle A_{p, 1} \cup A_{p, 2}\right\rangle$, that is $\operatorname{cod} E{\overline{F_{n_{p}}}}^{E} \leqslant \aleph_{0}$.

Let us suppose that none of the $L_{p}$ is barrelled. Then for each $p \in \mathbb{N}$ there is a barrel, say $T_{p}$, in $L_{p}$ which is not a neighbourhood of the origin in $L_{p}$. Now, since $\left\{L_{p} \cap E_{i}: p \in \mathbb{N}\right\}$ is an increasing sequence of subspaces of $E_{i}$ covering $E_{i}, i=1,2$, there must be some positive integer $q \in \mathbb{N}$ such that $L_{q} \cap E_{i}$ is barrelled. Therefore, setting $V_{q}:=\bar{T}_{q}^{E}, V_{q} \cap L_{q} \cap E_{i}$ is a neighbourhood of the origin in $L_{q} \cap E_{i}$.

On the other hand, $L_{q} \cap E_{i}$ is dense in $E_{i}$ since $\overline{L_{q} \cap E_{i}} E_{i} \supset\left\langle\overline{F_{n_{q}} \cap E_{i}} E_{i} \cup A_{q, i}\right\rangle=$ $E_{i}$. Therefore, ${\overline{V_{q} \cap L_{q} \cap E_{i}}}_{i}$ is a neighbourhood of the origin in $E_{i}, i=1,2$, and $V_{q}$ is a neighbourhood of the origin in $E$ since ${\overline{V_{q} \cap L_{q} \cap E_{1}}}^{E_{1}} \times{\overline{V_{q} \cap L_{q} \cap E_{2}}}^{E_{2}} \supset V_{q}+V_{q}=$ $2 V_{q}$. Hence $T_{q}$ is a neighbourhood of the origin in $L_{q}$, which is not possible.

In order to show that this result is true for arbitrarily many spaces we shall need [1, Theorem 2] and [2, Proposition 4]:

LEMMA 1. Let $\left\{E_{i}: i \in I\right\}$ be a family of spaces such that for every finite subset $H \subset I, E(H)$ is quasi-suprabarrelled. Then $E=\Pi\left\{E_{i}: \in I\right\}$ is quasi-suprabarrelled.

LEMMA 2. Let $\left\{E_{i}: i \in I\right\}$ be a family of spaces and $\mathcal{B}$ a countable family of closed absolutely convex subsets of $E=\Pi\left\{E_{i}: i \in I\right\}$ such that $\operatorname{cod}_{E}\langle B\rangle>\aleph_{0}$ for each $B \in \mathcal{B}$. Suppose that $\mathcal{F}:=\{\langle B\rangle: B \in \mathcal{B}\}$ covers $E$ and let $\mathcal{F}_{\boldsymbol{i}}:=\{F \in$ $\left.\mathcal{F}: \operatorname{cod}_{E(\{i\})} F \cap E(\{i\})>\aleph_{0}\right\}$. If for each $F \in \mathcal{F}$ there is a finite subset $J(F)$ of $I$ 
such that $F \supset E(I \backslash J(F))$, then there exists some $j \in I$ such that $\mathcal{F}_{j}$ covers $E(\{j\})$.

THEOREM 1. If $\left\{E_{i}: i \in I\right\}$ is a family of countably quasi-suprabarrelled spaces, then $E=\Pi\left\{E_{i}: i \in I\right\}$ is countably quasi-suprabarrelled.

Proof: By Lemma $1, E$ is quasi-suprabarrelled. So, if $E$ is not countably quasi-suprabarrelled, there exists an increasing sequence of barrelled subspaces of $E$ covering $E,\left\{F_{n}: n \in \mathbb{N}\right\}$, such that $\operatorname{cod}_{E}{\overline{F_{n}}}^{-E}>\dot{\aleph}_{0}$ for every $n \in \mathbb{N}$. Then $\left\{\overline{F_{n}}: n \in \mathbb{N}\right.$ and $\overline{F_{n}} \supset E\left(I \backslash J_{n}\right)$ with $J_{n}$ a finite subset of $\left.I\right\}$ is also an increasing sequence of barrelled subspaces of $E$ covering $E$, [12, Proposition 4].

Now, by Lemma 2, there exists some $j \in I$ such that $\left\{\overline{F_{n}}: n \in \mathbb{N}\right.$ and $\left.\operatorname{cod}_{E(\{j\})} \overline{F_{n}} \cap E(\{j\})>\aleph_{0}\right\}$ covers $E(\{j\})$, which is not possible since $E(\{j\})$ is countably quasi-suprabarrelled.

Finally let us recall that a locally convex space $E$ is a $\Gamma_{r}$-space if given any quasicomplete subspace $G$ of $E^{*}\left(\sigma\left(E^{*}, E\right)\right)$ such that $G \cap E^{\prime}$ is dense in $E^{\prime}\left(\sigma\left(E^{\prime}, E\right)\right)$, then $G$ contains $E^{\prime}$, and that $\Gamma_{r}$-spaces are the maximal class of locally convex spaces satisfying the closed graph theorem when barrelled spaces are considered as the domain, (see [8] and [10, Chapter 1, Section 6.2]). Moreover [8, Corolario 1.14] provides:

Lemma 3. Let $f$ be a continuous linear mapping from a barrelled space $E$ into $F$. If $F$ is a $\Gamma_{r}$-space then $f$ has a continuous extension from the completion of $E$ into $F$.

THEOREM 2. Let $E$ be a countably quasi-suprabarrelled space and suppose $\left\{F_{n}: n \in \mathbb{N}\right\}$ is an increasing sequence of subspaces of $F$ such that on each $F_{n}$ there exists a topology, $\tau_{n}$, finer than the original one so that $F_{n}\left(\tau_{n}\right)$ is a $\Gamma_{r}$-space. If $f$ is a linear mapping from $E$ into $F$ with closed graph then either there is some $p \in \mathbb{N}$ such that $f(E) \subset F_{p}$ and $f$ is continuous or there is a topological complement $H$ of $\varphi$ in $E$ such that $f(H) \subset F_{p}, f$ being continuous.

Proof: The sequence of subspaces $\left\{f^{-1}\left(F_{n}\right): n \in \mathbb{N}\right\}$ of $E$ is increasing and covers $E$, so there must to some $p \in \mathbb{N}$ such that $f^{-1}\left(F_{p}\right)$ is barrelled and its closure, $H$, has countable codimension in $E$. Let $L$ be a topological complement of $H$ in $E$. If $\operatorname{dim} L<\aleph_{0}$, then the restriction of $f$ to $L$ is continuous. If $\operatorname{dim} L=\aleph_{0}$, then $L \cong \varphi$ and the restriction of $f$ to $L$ is continuous, too. Thus in order to see that $f$ is continuous it is enough to show that the restriction $\left.f\right|_{H}$ of $f$ to $H$ is continuous. The restriction $g$ of $f$ to $f^{-1}\left(F_{p}\right)$ has closed graph in $f^{-1}\left(F_{p}\right) \times F_{p}\left(\tau_{p}\right)$ and thus is continuous. Now Lemma 3 allows us to extend $g$ to a continuous linear mapping $h: H \rightarrow F_{p}\left(\tau_{p}\right)$. Let us show that $h=\left.f\right|_{H}$. Given $x \in H$, let $\left\{x_{i}: i \in I\right\}$ be a net in $f^{-1}\left(F_{p}\right)$ converging to $x$ in $H$. Then the net $\left\{f\left(x_{i}\right): i \in I\right\}$ converges to $h(x)$ in $F_{p}\left(\tau_{p}\right)$ and, consequently, in $F$. Hence $f(x)=h(x)$ since $\left.f\right|_{H}$ has closed graph in $H \times F$. 
As $f(H) \subset F_{p}$, the proof is complete if $\operatorname{dim} L=\aleph_{0}$. If $\operatorname{dim} L<\aleph_{0}$, it is clear that there is some $q \in \mathbb{N}$ so that $f(L) \subset F_{q}$, and therefore $r=\max \{p, q\}$ gives $f(E) \subset F_{r}$.

\section{REFERENCES}

[1] J.C. Ferrando and M. López-Pellicer, 'Quasi-suprabarrelled spaces', J. Austral. Math. Soc. Ser. A 46 (1989), 137-145.

[2] J.C. Ferrando and J. Más, 'Quasi totally barrelled spaces', Portugal Math. 47 (1990), 205-213.

[3] J.C. Ferrando and L.M. Sánchez Ruiz, 'Algunos espacios tonelados de clase $\aleph_{0}$ ', in $A c-$ tas XV Jornadas Luso-Espanholas de Matematica, Vol II (Evora, Portugal, 1990), pp. 267-271.

[4] P. Pérez Carreras and J. Bonet, 'Remarks and examples concerning suprabarrelled and totally barrelled spaces', Arch. Math. 39 (1982), 340-347.

[5] W.J. Robertson, I. Tweddle and F.E. Yeomans, 'On the stability of barrelled topologies III', Bull. Austral. Math. Soc. 22 (1980), 99-112.

[6] S.A. Saxon, 'Nuclear and product spaces, Baire-like spaces and the strongest locally convex topology', Math. Ann. 197 (1972), 87-106.

[7] S.A. Saxon and M. Levin, 'Every countable-codimensional subspace of a barrelled space is barrelled', Proc. Amer. Math. Soc. 29 (1971), 91-96.

[8] M. Valdivia, 'Sobre el teorema de la gráfica cerrada', Collect. Math. 22 (1971), 51-72.

[9] M. Valdivia, 'On suprabarrelled spaces', in Func. Anal. Holomorphy and Approximation Theory. Rio de Janeiro 1978, Lecture Notes in Math. 843 (Springer-Verlag, Berlin, Heidelberg, New York, 1981), pp. 572-580.

[10] M. Valdivia, Topics in locally convex spaces, Math. Studies 67 (North Holland, Amsterdam, 1982).

[11] M. Valdivia, 'Three-space problems and projective tensor products of locally convex spaces', Proc. Royal Irish Acad. 86 A (1986), 127-141.

[12] M. Valdivia and P. Pérez Carreras, 'On totally barrelled spaces', Math. Z. 178 (1981), 263-269.

Departamento de Matemática Aplicada

Universidad Politécnica de Valencia

46071 Valencia

Spain 